\title{
COVID-19 and electrophysiology procedures—review, reset, reboot!!!
}

\author{
Naga Venkata K. Pothineni ${ }^{1}$ - Pasquale Santangeli ${ }^{1} \cdot$ Rajat Deo $^{1} \cdot$ Francis E. Marchlinski ${ }^{1} \cdot$ Matthew C. Hyman $^{1}$
}

Received: 9 July 2020 / Accepted: 10 September 2020 / Published online: 15 September 2020

(C) Springer Science+Business Media, LLC, part of Springer Nature 2020

The novel coronavirus (COVID-19) pandemic has created an unprecedented health care crisis leading to rationing and diversion of resources. While great focus has been placed on managing COVID-19 patients, clinicians must simultaneously balance caring for patients who are not actively infected. This dichotomy of thinking has become apparent in procedureladen disciplines including cardiac electrophysiology (EP). Providers have postponed many elective medical procedures with the goal of limiting unnecessary COVID-19 exposures to healthy individuals and to divert available resources to COVID-19 care. Although this shift away from elective procedures has been endorsed by major professional societies, it represents a fundamental change in how physicians provide routine/non-COVID-19-related care [1].

While it is generally accepted that the delivery of clinical care has changed in the last several months, there has been little quantification to what extent elective procedural care has been impacted. To address this question, we examined EP procedural volume in the Philadelphia region as a paradigm of how the COVID-19 outbreak has affected electrophysiologic care. Two major arrhythmia ablation vendors and two permanent pacemaker (PPM)/implantable cardioverter defibrillator (ICD) vendors were queried for their procedural volume from January 16 to April 30, 2020. Product vendors were used for data collection as they routinely track ablation/device patterns to provide technology support and could provide anonymized product utilization information. The Philadelphia region (11 counties) was selected for analysis as it has documented a significant number of COVID-19 cases and represents 10 teaching and 17 non-teaching hospitals. Ablation data and PPM/ICD data were pooled across vendors and hospitals in a bi-weekly fashion to generate a cross-section of procedural behavior. COVID-19 outbreak data in the Philadelphia region

Matthew C. Hyman

matthew.hyman@pennmedicine.upenn.edu

1 Section of Cardiac Electrophysiology, Division of Cardiovascular Medicine, Perelman School of Medicine, University of Pennsylvania, 9 Founders Pavilion, Philadelphia, PA 19104, USA were obtained from the Johns Hopkins Coronavirus Resource Center, https://coronavirus.jhu.edu/us-map.

COVID-19 first was identified in the Philadelphia area on March 7, 2020. Since this time, the number of EP procedures in the region has trended downward (Fig. 1). The cumulative prevalence of Philadelphia region COVID-19 was strongly, but inversely associated with regional EP procedure volume during the study period (Spearman rank correlation coefficient for arrhythmia ablation $(\rho=-0.82 ; P=0.02)$ and $\mathrm{PPM} / \mathrm{ICD}$ implantation $(\rho=-0.96 ; P<0.001))$. Arrhythmia ablation procedures decreased by $80 \%$ from a baseline of 781 procedures in the 6 weeks preceding the local COVID-19 outbreak to 158 in the subsequent 6 weeks. A greater reduction was seen in atrial fibrillation (AF) ablations (83\%) compared with ventricular tachycardia (VT) ablations (59\%) during this interval. Similarly, device implantations decreased by $47 \%$ ( 896 to 475) after the regional COVID-19 outbreak. Generator replacement and PPM and ICD procedures fell by $40 \%, 56 \%$, and $78 \%$ respectively.

A strong inverse relationship between regional EP procedure volume and the surge in COVID-19 cases that we observed is not surprising and is in accordance with societal and governmental recommendations. In addition to physician practice variations, patient avoidance of medical care may have also affected EP case volume similar to the reduction seen in ST-elevation myocardial infarction interventions [2]. Although overall procedural trends are consistent with the cardiology community's general adoption of taskforce recommendations to postpone elective medical procedures, categorization of cases as elective or urgent can be somewhat subjective. Our data does not contain data to examine the clinical parameters that defined procedural urgency, though trends of procedural subtypes suggest that VT ablations, pacemaker implantations, and generator changes were viewed as more urgent than AF ablations and ICD implantations.

While great effort and focus is appropriately being placed on COVID-19, the requirement for routine/non-COVID-19related care remains unchanged. Adoption of recommendations and adaptation to a new normal by the EP community 
Fig. 1 Relationship between electrophysiologic procedures and COVID-19 in the Philadelphia region. Arrhythmia ablation procedures (a) and permanent pacemaker (PPM) and implantable cardioverter defibrillator (ICD) procedures (b) are plotted over time in blue. Regional COVID-19 prevalence is plotted in red. Correlation coefficients were calculated using Spearman's $\rho$ a

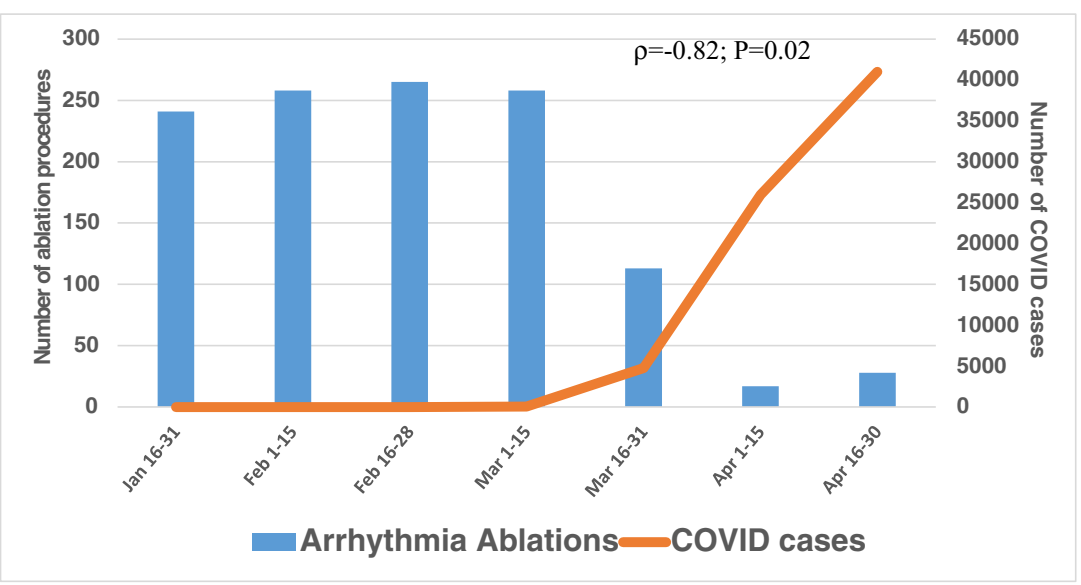

b

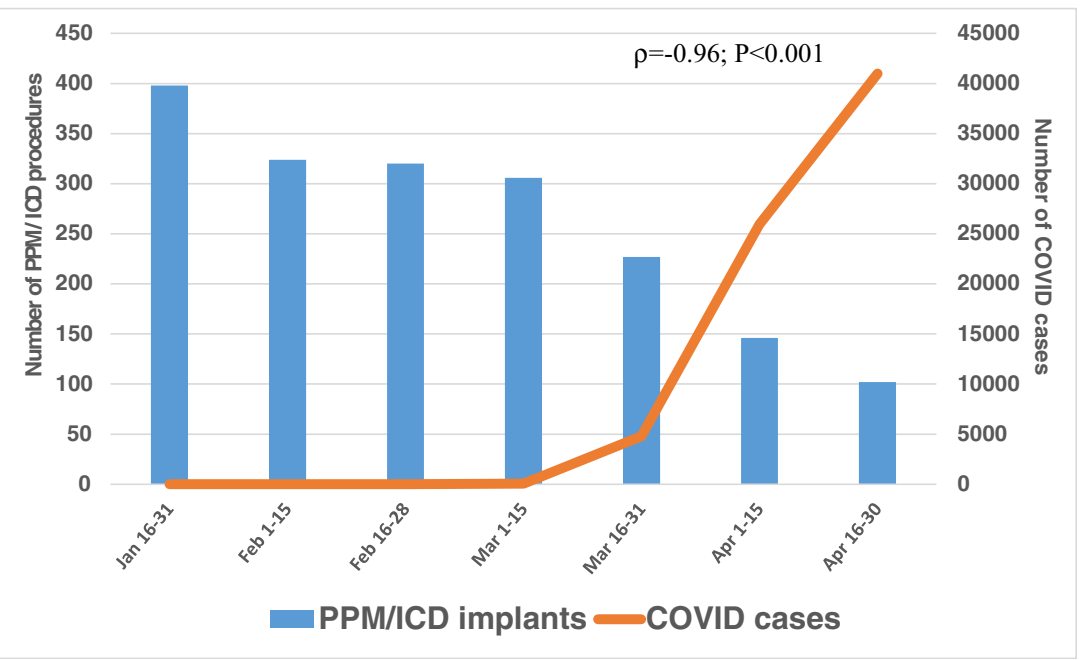

at large is commendable but still raises important questions. As we move towards a phase of procedural resurgence, how can we use this data to guide procedural planning? How do we continue to risk stratify procedural urgency while balancing symptom control and preventing unwanted hospitalizations? Conversely, if we are overly restrictive with non-COVID-19 care, how can we ensure that the postponement of EP procedures by both physician and patient decision-making does not exacerbate the high burden of non-COVID-related sudden cardiac death already seen in some countries? [3]. These questions become only more pertinent as secondary waves of COVID-19 are on the rise in many parts of the USA.

Unfortunately, we do not have answers for all these questions. Lessons learned from examining Philadelphia procedural trends suggest that while more urgent procedures like VT ablations and pacemakers have been less impacted, atrial fibrillation ablations and primary prevention ICDs may experience a significant delay until COVID-19 is better contained. To counteract this problem, at the University of Pennsylvania, we have begun to expand procedural scheduling while simultaneously implementing universal masking protocols, limitations on the number of peri-procedural visitors, pre-procedural COVID-19 screening, avoidance of aerosolizing procedures like high-frequency low-tidal volume ventilation during ablative procedures, and limiting the proximity of EP lab personnel during intubation and extubation. In addition, a concerted effort was made towards enrolling all device patients in remote monitoring to facilitate effective telemedicine care and to prioritize procedural availability and urgency. Using these techniques, we have successfully performed 565 EP procedures without documented COVID-19 infection of either a patient or staff member. Consistent with national trends, at the regional peak of COVID-19, elective procedures like mildly symptomatic atrial fibrillation were transiently deferred in favor of more urgent procedures like ventricular tachycardia storm. Given our initial success and safety, we have begun to reexpand our clinical efforts to include more elective cases. Further studies will be needed to determine if these 
interventions are sufficient, excessive or inadequate, but for our hospital, these have been our first steps in expanding care to all of our EP patients.

We are only beginning to understand the long-term implications of COVID-19 and the profound limitations it has placed on the physician's ability to deliver medical care. As we and others try to practice in this challenging clinical environment, our old ways of doing things may no longer suffice and new safety protocols will need be developed. Only then can we as physicians successfully plan for re-expansion of services as our patients' need for medical care has not been diminished; it has merely been postponed.

\section{References}

1. Lakkireddy DR, Chung MK, Gopinathannair R, Patton KK, Gluckman TJ, Turagam M, Cheung J, Patel P, Sotomonte J,
Lampert R, Han JK, Rajagopalan B, Eckhardt L, Joglar J, Sandau K, Olshansky B, Wan E, Noseworthy PA, Leal M, Kaufman E, Gutierrez A, Marine JM, Wang PJ and Russo AM. Guidance for cardiac electrophysiology during the coronavirus (COVID-19) pandemic from the Heart Rhythm Society COVID-19 Task Force; Electrophysiology Section of the American College of Cardiology; and the Electrocardiography and Arrhythmias Committee of the Council on Clinical Cardiology, American Heart Association. Circulation. 2020.

2. Garcia S, Albaghdadi MS, Meraj PM, Schmidt C, Garberich R, Jaffer FA, et al. Reduction in ST-segment elevation cardiac catheterization laboratory activations in the United States during COVID-19 pandemic. J Am Coll Cardiol. 2020;75:2871-2.

3. Baldi E, Sechi GM, Mare C, Canevari F, Brancaglione A, Primi R, et al. Out-of-hospital cardiac arrest during the COVID-19 outbreak in Italy. N Engl J Med. 2020;383:496-8.

Publisher's note Springer Nature remains neutral with regard to jurisdictional claims in published maps and institutional affiliations. 